\title{
Aleitamento materno exclusivo em bebês com fissura de lábio e/ou palato
}

\section{Exclusive breastfeeding in infants with cleft lip and/or palate}

\author{
Camila Queiroz de Moraes Silveira Di Ninno ${ }^{1}$, Danila Moura ${ }^{1}$, Regina Raciff ${ }^{1}$, Sandra Valéria Machado ${ }^{1}$, \\ Christiane Marize Garcia Rocha², Rocksane de Carvalho Norton ${ }^{3}$, Fernanda Abalen Dias Martins ${ }^{1}$, \\ Denise Brandão de Oliveira e Britto ${ }^{4}$
}

\begin{abstract}
RESUMO
Objetivo: Investigar o aleitamento materno exclusivo em bebês com fissura de lábio e/ou palato e sua associação com o tipo de fissura. Métodos: Foram coletados dados de 137 prontuários, obtidos pela pediatra na primeira consulta. A amostra constituiu-se das fichas de pediatria de bebês de ambos os gêneros, com fissura de lábio e/ou palato, nascidos a termo e sem outras anomalias associadas, que chegaram para a primeira consulta entre zero e 12 meses. Os dados foram analisados estatisticamente. Resultados: Houve predomínio da fissura transforame incisivo em bebês do gênero masculino. Os bebês apresentavam média de idade de 53 dias e mediana de 33 dias na primeira consulta. Quanto ao aleitamento materno exclusivo, este ocorreu em 7,3\% do total da amostra, sendo 6,57\% no grupo pré-forame incisivo e $0,73 \%$ no grupo pós-forame incisivo, mas não ocorreu no grupo transforame incisivo. Houve associação significativa entre o tipo de fissura e o aleitamento. Conclusão: $\mathrm{O}$ aleitamento exclusivo está presente em pequena parte da amostra total de bebês com fissura de lábio e/ou palato, sendo frequente na fissura pré-forame incisivo.
\end{abstract}

Descritores: Fenda labial; Fissura palatina; Recém-nascido; Alimentação; Aleitamento materno; Nutrição enteral

\section{INTRODUÇÃO}

O melhor alimento para o bebê é o leite materno, uma vez que possui nutrientes, fatores de proteção para o lactente contra infecções e fatores que favorecem o seu crescimento e o desenvolvimento do sistema imunológico ${ }^{(1)}$. Além disso, o aleitamento no seio materno é fundamental para o desenvolvimento e a maturação da musculatura orofacial, proporcionando boa oclusão, melhor relação entre maxila e mandíbula, expansão dos seios maxilares, vedamento labial, conservação da respiração nasal, posição lingual adequada e diminuindo a incidência de cáries ${ }^{(2)}$.

Trabalho realizado no Curso de Fonoaudiologia, Pontifícia Universidade Católica de Minas Gerais - PUC Minas - Belo Horizonte (MG), Brasil.

(1) Curso de Fonoaudiologia, Pontifícia Universidade Católica de Minas Gerais - PUC Minas - Belo Horizonte (MG), Brasil.

(2) Centro de Tratamento e Reabilitação de Fissuras Labiopalatinas e Deformidades Craniofaciais - PUC Minas/Hospital da Baleia - Belo Horizonte (MG), Brasil.

(3) Faculdade de Medicina, Universidade Federal de Minas Gerais - UFMG - Belo Horizonte (MG), Brasil.

(4) Programa de Pós-graduação (Doutorado) em Letras, Pontifícia Universidade Católica de Minas Gerais - PUC Minas - Belo Horizonte (MG), Brasil; Curso de Fonoaudiologia, Pontifícia Universidade Católica de Minas Gerais - PUC Minas - Belo Horizonte (MG), Brasil.

Endereço para correspondência: Camila Queiroz de Moraes Silveira Di Ninno. Av. Dom José Gaspar, 500 - Prédio 25, Coração Eucarístico, Belo Horizonte (MG), Brasil, CEP: 30535-610. E-mail: camilaninno@uol.com.br Recebido em: 28/7/2010; Aceito em: 24/3/2011
$\mathrm{Na}$ literatura ${ }^{(2-7)}$, vários fatores são apontados como responsáveis pelo sucesso do aleitamento materno exclusivo, os quais serão descritos a seguir.

O recém-nascido apresenta reflexos fundamentais para sua sobrevivência e dentre eles, três são essenciais para a amamentação $0^{(2)}$ :

- Reflexo de busca: quando a mãe toca a face do bebê com o bico da mama ou com o dedo, o bebê vira a cabeça para o lado de onde veio o estímulo e abre a boca;

- Reflexo de sucção: quando o bico da mama toca o palato, o bebê começa a sugar;

- Reflexo de deglutição: estando com a boca cheia de leite, o bebê deglute espontaneamente.

Além dos reflexos, para que haja sucesso no aleitamento materno, antes de tudo, é importante que a mama esteja íntegra e desenvolvida ${ }^{(3)}$. Para que haja um aleitamento eficaz é necessário que o bebê esvazie os seios lactíferos, o que significa que ele precisa introduzir a maior parte da aréola na boca. Durante a sucção, o bico da mama aumenta em três vezes o seu comprimento dentro da cavidade oral do bebê, encostando-se no palato duro e indo até o palato mole, estimulando a sucção. Neste momento, a língua se desloca anteroposteriormente, executando um movimento ondulatório denominado onda peristáltica. Estes movimentos, realizados por cerca de vinte músculos orofaciais diferentes, resultam na ejeção do leite dos seios lactíferos para dentro da cavidade oral do lactente ${ }^{(4)}$.

Outro fator que influencia diretamente no sucesso da 
amamentação é o modo de realizar esta ação. A escolha de um local que proporcione mais conforto e tranquilidade para a mãe é fundamental, pois favorece uma boa mamada ${ }^{(1)}$. Assim, a posição adequada para a amamentação será aquela que traga maior conforto à mãe, desde que o bebê não fique em uma posição deitada ${ }^{(2-5)}$.

Outro ponto que deve ser abordado em relação ao aleitamento materno exclusivo é o vínculo que o mesmo propicia entre a mãe e o bebê. A amamentação proporciona uma interrelação, que constrói o vínculo mãe-bebê, sendo primordial para o psiquismo humano, justamente por estas trocas que acontecem durante o período. Este sucesso só é possível pelo prazer da mãe em realizar sua tarefa de amamentar e pelo prazer do bebê em sugar. Quando a amamentação decorre sem dificuldades, a criança encontra meios nutritivos e psicoemocionais para seu crescimento e desenvolvimento ${ }^{(5,6)}$.

Retomando a discussão quanto às vantagens do aleitamento materno, enfocaremos os distúrbios da motricidade oromiofuncional, provenientes da ausência ou ineficácia do mesmo.

As disfunções orais mais frequentes decorrentes dos impedimentos da amamentação são a respiração oral, a tonicidade diminuída dos músculos orofaciais, as mal oclusões dentárias, movimentos inadequados da mandíbula e postura inadequada da língua, interferindo na mastigação, na deglutição e na fala ${ }^{(7)}$.

No entanto, há situações em que o aleitamento materno exclusivo não é possível, como nos casos de bebês filhos de mães com o vírus da imunodeficiência humana (HIV positivo), bebês com comprometimento neurológico e malformações craniofaciais. Dentre as malformações craniofaciais, as mais frequentes são as fissuras congênitas de lábio e/ou palato presentes em um a cada 650 bebês nascidos vivos ${ }^{(8)}$.

A dificuldade de alimentação de bebês com fissura surge logo após o nascimento, devido ao prejuízo no mecanismo de sucção e deglutição, decorrente da falta de integridade das estruturas anatômicas. Nesta fase inicial, a prioridade é a nutrição do bebêe o acompanhamento de seu ganho de peso por um pediatra deve ser frequente ${ }^{(9-13)}$.

As primeiras orientações aos pais de bebês com fissura devem ser dadas em relação às implicações imediatas da fissura, em especial na alimentação; à importância de um tratamento especializado, com indicação de centros e profissionais; e à possibilidade, em se tratando de uma fissura isolada, de a criança vir a se desenvolver normalmente ${ }^{(10)}$.

Quanto à alimentação, as orientações fonoaudiológicas às mães de bebês com fissura de lábio e/ou palato deve abordar a importância do aleitamento materno direto ou, se necessário, ordenhado e oferecido em mamadeira, com bicos macios de látex e furo ligeiramente aumentado. Deve-se também considerar que a mamada destes bebês é mais demorada em virtude da menor força de sucção; que o lado da fissura não deve ser evitado, a fim de estimular a musculatura, e que o bebê deve ser mantido em posição mais ereta, para evitar o refluxo nasal de leite e a penetração do mesmo no conduto auditivo. Além disso, devido à ingestão excessiva de ar no momento da alimentação, deve-se fazer mais pausas para propiciar a eructação ${ }^{(9-12)}$.

Como resultado das dificuldades alimentares, bebês com fissura geralmente apresentam ingesta com volume reduzido, tempo prolongado e intervalos pequenos, podendo não apre- sentar ganho de peso adequado ou, inclusive, vir a perder peso, em especial nas fissuras do tipo transforame e pós-forame incisivo $^{(11,14,15)}$

Sabendo-se da importância do aleitamento materno e conhecendo-se as dificuldades de alimentação inerentes à fissura de lábio e/ou palato, o objetivo deste estudo foi investigar o aleitamento materno exclusivo em bebês com fissura de lábio e/ou palato e sua associação com o tipo de fissura.

\section{MÉTODOS}

Este estudo, aprovado pelo Comitê de Ética em Pesquisa da Pontifícia Universidade Católica de Minas Gerais (PUC Minas) sob o número CAAE - 0323.0.213.007-07; foi baseado na coleta de dados nos arquivos do setor de pediatria do Centro de Tratamento e Reabilitação de Fissuras Labiopalatais e Deformidades Craniofaciais vinculado à Pontifícia Universidade Católica de Minas Gerais.

A amostra deste estudo foi constituída por 137 fichas de pacientes com fissura de lábio e/ou palato do Centro de Tratamento. Como critérios de inclusão, foram selecionadas fichas de bebês de ambos os gêneros, com fissura de lábio e/ou palato, nascidos a termo e que chegaram para a primeira consulta no setor de pediatria com idade entre zero e 12 meses. Foram excluídos os casos que apresentavam síndromes, cardiopatias e comprometimento do sistema nervoso central, associados.

Foram coletados dados relativos ao gênero, tipo de fissura diagnosticada, tempo de aleitamento materno exclusivo, aleitamento por outras vias, tipo de maternidade (particular ou pública) e a cidade de origem, com protocolo para coleta dos dados desenvolvido para o presente estudo (Anexo 1).

A classificação das fissuras utilizada neste estudo foi a proposta por Spina et $\mathrm{al}^{(16)}$, por ser a mais utilizada no Brasil. Esta classificação tem como ponto de referência anatômica o forame incisivo que separa os palatos primário e secundário e divide as fissuras em quatro diferentes grupos: Grupo I - fissura pré-forame incisivo, Grupo II - fissura transforame incisivo, Grupo III - fissura pós-forame incisivo e Grupo IV - fissuras raras da face.

Após a tabulação dos dados, estes foram analisados estatisticamente por meio dos testes Qui-quadrado e Exato de Fisher, com nível de significância de $5 \%(\mathrm{p} \leq 0,05)$.

\section{RESULTADOS}

Do total da amostra, houve predomínio da fissura transforame incisivo e do gênero masculino (Tabela 1). Os bebês

Tabela 1. Caracterização da amostra por gênero e tipo de fissura

\begin{tabular}{lccc}
\hline \multirow{2}{*}{ Tipo de fissura } & \multicolumn{2}{c}{ Gênero } & \multirow{2}{*}{ Total $(\%)$} \\
\cline { 2 - 3 } & Feminino (\%) & Masculino $(\%)$ & \\
\hline Pré-forame incisivo & $4,38(n=6)$ & $9,49(n=13)$ & $13,87(n=19)$ \\
Transforame incisivo & $16,79(n=23)$ & $34,30(n=47)$ & $51,09(n=70)$ \\
Pós-forame incisivo & $18,24(n=25)$ & $16,79(n=23)$ & $35,03(n=48)$ \\
Total & $39,41(n=54)$ & $60,59(n=83)$ & $100(n=137)$ \\
\hline
\end{tabular}

Teste de Qui-quadrado $(p=0,083)$ 
Tabela 2. Comparação entre o tipo de fissura e naturalidade

\begin{tabular}{lcccc}
\hline Tipo de fissura & \multicolumn{3}{c}{ Naturalidade } & Total $(\%)$ \\
\cline { 2 - 4 } & Belo Horizonte $(\%)$ & RMBH $(\%)$ & Interior $(\%)$ & $13,87(\mathrm{n}=19)$ \\
Pré-forame incisivo & $8,03(\mathrm{n}=11)$ & $2,92(\mathrm{n}=4)$ & $2,92(\mathrm{n}=4)$ & $51,10(\mathrm{n}=70)$ \\
Transforame incisivo & $33,58(\mathrm{n}=46)$ & $6,60(\mathrm{n}=9)$ & $10,95(\mathrm{n}=15)$ & $35,03(\mathrm{n}=48)$ \\
Pós-forame incisivo & $18,25(\mathrm{n}=25)$ & $5,84(\mathrm{n}=8)$ & $10,95(\mathrm{n}=15)$ & $100(\mathrm{n}=137)$ \\
Total & $59,86(\mathrm{n}=82)$ & $15,36(\mathrm{n}=21)$ & $24,82(\mathrm{n}=34)$ & \\
\hline
\end{tabular}

Teste Exato de Fisher $(p=0,560)$

Legenda: $\mathrm{RMBH}=$ Região Metropolitana de Belo Horizonte

apresentavam média de idade de 53 dias e mediana de 33 dias na data da primeira consulta.

Dos bebês analisados, a maioria nasceu em maternidades públicas da cidade de Belo Horizonte (Tabela 2).

De acordo com os resultados do teste de Qui-quadrado e teste Exato de Fisher, não houve diferença entre os diferentes tipos de fissura em relação ao gênero, nem em relação à naturalidade do bebê.

Em relação ao aleitamento materno, considerando o total da amostra, apenas 7,3\% dos bebês receberam amamentação exclusiva, sendo que a maioria tinha fissura pré-forame incisivo (Tabela 3). Nos casos de fissura pré-forame incisivo $(n=10)$, sete deles utilizaram mamadeira e três utilizaram conta gotas, copinho e/ou colher; na fissura transforame incisivo $(n=70), 47$ deles receberam o leite materno na mamadeira, colher, copo ou sonda nasogástrica, um recebeu outro tipo de leite pela seringa, um por conta gotas e 21 pela mamadeira e, na fissura pós-forame incisivo $(n=47)$, todos faziam uso de mamadeira, colher, copo ou sonda nasogástrica (SNG).

Tabela 3. Caracterização da amostra por tipo de fissura e aleitamento materno exclusivo

\begin{tabular}{lccc}
\hline \multirow{2}{*}{ Tipo de fissura } & \multicolumn{2}{c}{ Aleitamento exclusivo } & \multirow{2}{*}{ Total $(\%)$} \\
\cline { 2 - 3 } & \multicolumn{1}{c}{$\operatorname{Sim}(\%)$} & Não $(\%)$ & \\
\hline Pré-forame incisivo & $6,57(\mathrm{n}=9)$ & $7,30(\mathrm{n}=10)$ & $13,87(\mathrm{n}=19)$ \\
Transforame incisivo & $0,00(\mathrm{n}=0)$ & $51,10(\mathrm{n}=70)$ & $51,10(\mathrm{n}=70)$ \\
Pós-forame incisivo & $0,73(\mathrm{n}=1)$ & $34,30(\mathrm{n}=47)$ & $35,03(\mathrm{n}=48)$ \\
Total & $7,30(\mathrm{n}=10)$ & $92,70(\mathrm{n}=127)$ & $100(\mathrm{n}=137)$ \\
\hline
\end{tabular}

Teste Exato de Fisher $(p=0,000)$

O teste exato de Fisher encontrou associação entre o tipo de fissura e o aleitamento materno exclusivo, sendo este mais frequente em bebês com fissura do tipo pré-forame, ou seja, sem o comprometimento do palato.

Dos dez bebês que receberam amamentação exclusiva, a maioria (70\%) era do gênero masculino, todos nascidos em maternidades públicas, sendo metade deles $(50 \%)$ na cidade de Belo Horizonte.

\section{DISCUSSÃO}

$\mathrm{Na}$ amostra analisada houve maior prevalência da fissura do tipo transforame e do gênero masculino, o que também foi encontrado em outras populações de indivíduos com fis$\operatorname{sura}^{(8,11,13,14,17-19)}$.

Neste estudo houve associação significativa entre o tipo de fissura e o aleitamento materno exclusivo. Dos bebês que tiveram aleitamento materno exclusivo, a grande maioria apresentava fissura pré-forame incisivo. Este achado era esperado, pois se sabe que bebês com fissura labial isolada apresentam menores dificuldades para se alimentar, o que é justificado pela integridade do palato ${ }^{(11,12,14,15,18,20-22)}$.

Bebês com outros tipos de fissura também fizeram uso do leite materno, mas principalmente por meio da ordenha e, muitas vezes, associado ao uso de outros tipos de leite. $\mathrm{Na}$ fissura de lábio, a integridade do palato proporciona o desenvolvimento de pressão intraoral negativa e positiva, necessárias para a realização da sucção, o que não ocorre quando o palato encontra-se aberto pela presença de fissura ${ }^{(21,22)}$. Neste caso, a comunicação entre as cavidades oral e nasal dificulta a formação de pressão intraoral.

Por desconhecimento ou medo de alimentar o bebê com fissura, devido à falta de orientações adequadas às famílias ao nascimento ${ }^{(10,11,23)}$, muitas vezes são oferecidos volumes pequenos, em mamadeiras com bicos de orifícios inadequados e não são utilizadas técnicas facilitadoras de amamentação. Tudo isto prolonga o tempo de mamada e o gasto energético do bebê, dificultando seu desempenho alimentar ${ }^{(11)}$.

Em bebês com fissura de palato, o uso de utensílios que facilitem a ingesta de leite com menor gasto calórico, ou seja, o uso de mamadeiras com bico de látex, colheres ou copinhos, pode ser importante para o maior ganho de peso nesse grupo e deve ser mais investigado em futuros estudos.

No geral, o número de bebês em amamentação exclusiva foi baixo, mas provavelmente influenciado por uma amostra pequena de bebês com fissura pré-forame. Na literatura há autores que, ao compararem bebês com e sem fissura, encontraram um tempo menor de aleitamento materno no grupo com fissura ${ }^{(23)}$.

Deve-se ainda ressaltar que parte da amostra analisada (23\%) fez uso da SNG. Embora o motivo para isso não tenha sido averiguado neste estudo, acredita-se que nenhum destes bebês tivesse a real necessidade deste recurso, uma vez que foram excluídos os casos de prematuridade, síndromes, cardiopatias ou comprometimentos do sistema nervoso central $^{(13,15,19,22-24)}$. A indicação excessiva de SNG pode ter prejudicado o aleitamento no seio materno e evidenciou a falta de conhecimento por parte dos profissionais da área de saúde em relação à alimentação de bebês com fissura e aos recursos que podem ser utilizados para evitar o uso dessa alternativa. Este fato confirma a importância de orientação aos profissionais da saúde de maternidades, já apontado em estudos anteriores ${ }^{(10,13,20,23,24)}$. 
O fato de a maioria dos bebês em aleitamento materno exclusivo ter nascido em maternidades públicas pode estar relacionado ao maior incentivo ao aleitamento materno nestas instituições e/ou com questões econômicas da família.

Espera-se que este estudo possa contribuir para as orientações alimentares de bebês nascidos com fissura de lábio e/ ou palato, tanto nas maternidades, como nos centros especializados.

\section{CONCLUSÃO}

$\mathrm{O}$ aleitamento materno exclusivo estava presente em pequena parte da amostra total de bebês com fissura de lábio e/ou palato que participaram do presente estudo, sendo prevalente na fissura pré-forame incisivo.

\begin{abstract}
Purpose: To investigate exclusive breastfeeding in infants with cleft lip and/or palate and its association with the type of cleft. Methods: Data recorded by the pediatrician during the first consultation were obtained from 137 clinical charts. The sample consisted of the pediatric records of infants of both genders, with cleft lip and/or palate, born full term and without any other associated anomalies, who arrived for the first consultation between zero and 12 months of age. Data were statistically analyzed. Results: There was predominance of complete cleft lip and palate in male infants. Subjects' mean age was 53 days, with median of 33 days on the date of first consultation. Exclusive breastfeeding occurred in $7.3 \%$ of the total sample $-6.57 \%$ in cleft lip group, $0.73 \%$ in the cleft palate, and none in the complete cleft lip and palate group. There was significant association between type of cleft and breastfeeding. Conclusion: Exclusive breastfeeding is present in only a small part of the total sample of infants with cleft lip and/or palate, and is frequent in infants with incomplete cleft lip.
\end{abstract}

Keywords: Cleft lip; Cleft palate; Infant, newborn; Feeding; Breast feeding; Enteral nutrition

\section{REFERÊNCIAS}

1. Campiotto AR, Caretta VC. A influência do aleitamento materno e da alimentação sólida no padrão de mastigação. Fono Atual. 2003;6(25):13-8.

2. Vinha VH. O livro da amamentação. São Paulo: CLR Brasileiro; 1999.

3. Carvalho GO. S.O.S. respirador bucal: uma visão funcional e clínica da amamentação. São Paulo: Lovise; 2003.

4. Langes S. Aleitamento do lactente: cuidados especiais. São Paulo: Santos; 1999.

5. Pamplona V. Parto e psicodrama. São Paulo: Agora; 1990. Aspectos psicológicos na lactação; p.166-75.

6. Fontoura T. Aleitamento materno: uma perspectiva psicológica. Rev Téc-Cient Enferm. 2004;2(10):224-8.

7. Neiva FC, Cattoni DM, Ramos JL, Issler H. Desmame precoce: implicações para o desenvolvimento motor oral. J Pediatr (Rio J). 2003;79(1):7-12.

8. Nagem Filho H, Moraes N, Rocha RGF. Contribuição para o estudo da prevalência das más formações congênitas lábio-palatais na população escolar de Bauru. Rev Fac Odontol Univ São Paulo. 1968;6(2):111-28.

9. Di Ninno CQ. Abordagem fonoaudiológica atual nas fissuras labiopalatina. In: Britto AT, organizador. Livro de fonoaudiologia. São José dos Campos: Pulso; 2005. p. 325-38.

10. Di Ninno CQMS, Gomes R, Santos P, Bueno M, Galvão D, Meira A, et al. O conhecimento dos profissionais da área da saúde sobre fissura labiopalatina. Rev Soc Bras Fonoaudiol. 2004;9(2):93-101.

11. Rocha CM. Resultados da intervenção interdisciplinar precoce em crianças com fissura labiopalatal atendidas no centro de tratamento de fissuras [dissertação]. Minas Gerais: Universidade Federal Minas Gerais; 2008 .

12. Silva EB, Furia CLB, Di Ninno CQMS. Aleitamento em recém nascidos portadores de fissuras labiopalatina: dificuldade e métodos utilizados. Rev CEFAC. 2005;7(1):50-4

13. Amstalden-Mendes LG, Magna LA, Gil-da-Silva-Lopes VL. Neonatal care of infants with cleft lip and/or palate: feeding orientation and evolution of weight gain in a nonspecialized brazilian hospital. Cleft Palate Craniofac J. 2007;44(3):329-34.

14. Montagnoli LC, Barbieri MA, Bettiol H, Marques IL, Souza L. Prejuízo no crescimento de crianças com diferentes tipos de fissura lábio-palatina nos 2 primeiros anos de idade: um estudo transversal. J Pediatr (Rio J). 2005;81(6):461-5.

15. Reid J, Kilpatrick N, Reilly S. A prospective, longitudinal study of feeding skills in a cohort of babies with cleft conditions. Cleft Palate Craniofac J. 2006;43(6):702-9.

16. Spina V, Psillakis JM, Lapa FS, Ferreira MC. Classificação das fissuras labiopalatinas, sugestão de modificação. Rev Hosp Fac Med Univ São Paulo. 1972;27(1):5-6.

17. Masarei AG, Sell D, Habel A, Mars M, Sommerlad BC, Wade A. The nature of feeding in infants with unrepaired cleft lip and/or palate compared with healthy noncleft infants. Cleft Palate Craniofac J. 2007;44(3):321-8.

18. Campillay PL, Delgado SE, Brescovici SM. Avaliação da alimentação em crianças com fissura de lábio e/ou palato atendidas em um hospital de Porto Alegre. Rev CEFAC. 2010;12(2):257-66.

19. Di Ninno CQ, Fonseca LF, Pimenta MV, Vieira ZG, Fonseca JA, Miranda IC, et al. Levantamento epidemiológico dos pacientes portadores de fissura de lábio e/ou palato de um centro especializado de Belo Horizonte. Rev Cefac [Internet]. 2011 [citado 2011 Set 20]. Disponível em: http://www.scielo.br/pdf/rcefac/2011 nahead/100-10.pdf .

20. Oliver RG, Jones G. Neonatal feeding of infants born with cleft lip and/ or palate: parental perceptions of their experience in South Wales. Cleft Palate Craniofac J. 1997;34(6):526-32.

21. Reid J, Reilly S, Kilpatrick N. Sucking performance of babies with cleft conditions. Cleft Palate Craniofac J. 2007;44(3):312-20.

22. Silva EB, Rocha CM, Lage RR. O bebê com fissura labiopalatina: intervenção interdisciplinar. In: Jesus MS, Di Ninno CQ. Fissura labiopalatina: fundamentos para a prática fonoaudiológica. São Paulo: Roca; 2009. p. 10-28.

23. Smedegaard LH, Marxen DR, Moes JH, Glassou EN, Scientsan C. Hospitalization, breast-milk feeding, and growth in infants with cleft palate and cleft lip and palate born in Denmark. Cleft Palate Craniofac J. 2008;45(6):628-32.

24. Di Ninno CQ, Vieira FC, Lemos AM, Silva LF, Rocha CM, Norton RC, et al. A prevalência do uso de sonda nasogástrica em bebês portadores de fissura de lábio e/ou palato. Rev Soc Bras Fonoaudiol. 2010;15(4):578-83. 
Anexo 1. Protocolo de coleta de dados

Noprotocolo:

Gênero: ( ) Feminino ( ) Masculino

DN:

Idade na $1^{\text {a }}$ consulta:

meses dias

Maternidade:
( ) Pública
( ) Particular

Cidade:
( ) Belo Horizonte
( ) Região metropolitana de BH
( ) Interior MG

Alimentação:
( ) Aleitamento materno
( ) Mamadeira
( ) Chuquinha
( ) Copo ( ) Conta gota
( ) Colher
( ) Seringa
( ) Outros
( ) SNG

Tipo de leite:

( ) Materno ( ) Artificial especificar:

Fissura:
( ) Pré-forame
( ) Completa
( ) Incompleta
( ) D ( ) E ( ) Bilateral
( ) D ( ) E ( ) Bilateral
( ) D ( ) E ( ) Bilateral
( ) D
( ) Incompleta
( ) Bilateral
( ) Pós-forame
( ) D
( ) E 